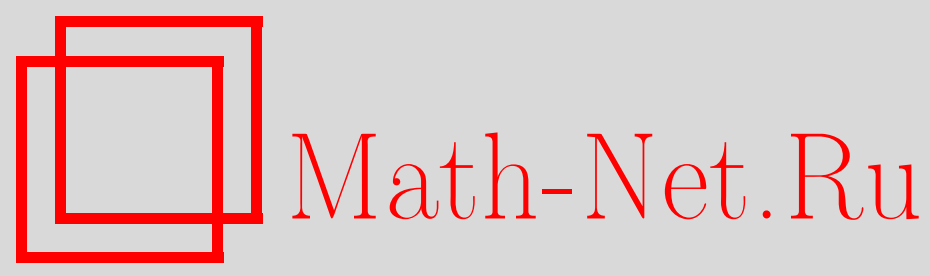

Е. С. Волкова, Аксиома Ф-голоморфных плоскостей для нормальных многообразий киллингова типа, Матем. заметки, 2002, том 71, выпуск 3, 364-372

DOI: https://doi.org/10.4213/mzm352

Использование Общероссийского математического портала Math-Net.Ru подразумевает, что вы прочитали и согласны с пользовательским соглашением http://www . mathnet.ru/rus/agreement

Параметры загрузки:

IP: 34.229 .108 .108

26 апреля 2023 г., $17: 23: 41$

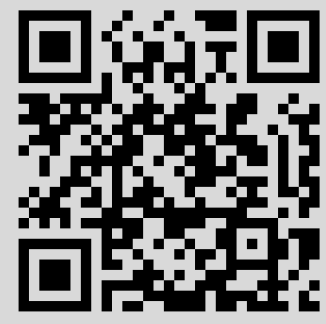




\title{
АКСИОМА Ф-ГОЛОМОРФНЫХ ПЛОСКОСТЕЙ ДЛЯ НОРМАЛЬНЫХ МНОГООБРАЗИЙ КИЛЛИНГОВА ТИПА
}

\author{
Е. С. Волкова
}

Изучаются свойства $\Phi$-голоморфной секционной кривизны нормальных многообразий киллингова типа, удовлетворяющих аксиоме $\Phi$-голоморфных плоскостей.

Библиография: 15 названий.

В последние десятилетия особым интересом геометров пользуются почти контактные метрические многообразия. Это объясняется не только богатой геометрий этих многообразий, но и их многочисленными приложениями в различньх областях математики и теоретической физики.

Если проанализировать свойства почти контактных метрических структур, можно заметить, что у многих из них (таких, как квазисасакиевых, сасакиевых, косимплектических) структурньй ковектор является киллинговой формой. Поэтому естественно возникает идея рассмотрения почти контактных метрических структур, для которых это свойство является определяющим. Примером такой структуры является нормальная структура киллингова типа (короче, $\mathscr{C} \mathscr{N} \mathscr{K}$-структура).

Одними из наиболее актуальных вопросов почти контактной геометрии являются изучение почти контактных многообразий, удовлетворяющих аксиоме $\Phi$-голоморфных плоскостей [1], а также исследование почти контактных многообразий точечно постоянной $\Phi$-голоморфной секционной кривизны [2].

Хорошо известно [3], что для сасакиевых и косимплектических многообразий выполнение аксиомы $\Phi$-голоморфных плоскостей эквивалентно точечному постоянству их $\Phi$-голоморфной секционной кривизны. Естественно возникает вопрос об аналогах этих результатов в случае $\mathscr{C} \mathscr{N} \mathscr{K}$-многообразий. Ответу на этот вопрос посвящена наша работа.

Пусть $M$ - гладкое многообразие размерности $2 n+1$.

ОПРЕДЕЛЕНИЕ 1 [1]. Почти контактной метрической структурой на многообразии $M$ называется совокупность $\{\Phi, \xi, \eta, g\}$ тензорных полей на $M$, где $g=\langle\cdot, \cdot\rangle-$ (псевдо) риманова метрика, $\Phi$ - тензор типа (1.1), называемый структурныл оператором, $\xi$-вектор, $\eta$-ковектор, называемые структурными вектором и ковектором соответственно. При этом
1) $\Phi(\xi)=0$;
2) $\eta \circ \Phi=0$;
3) $\eta(\xi)=1$;
4) $\Phi^{2}=-\mathrm{id}+\xi \otimes \eta$ 
5) $\langle\Phi X, \Phi Y\rangle=\langle X, Y\rangle-\eta(X) \eta(Y), X, Y \in \varkappa(M)$, где $\varkappa(M)$ - алгебра Ли гладких векторных полей на многообразии;

$d$ - оператор внешнего дифференцирования, $\nabla$ - риманова связность, $C^{\infty}(M)$ - алгебра гладких функций многообразия $M$. Все многообразия, тензорные поля и т.п. объекты предполагаются гладкими класса $C^{\infty}$.

Напомним [4], что почти контактная метрическая структура называется нормальной, если $N+\frac{1}{2} \xi \otimes d \eta=0$, где $4 N(X, Y)=[\Phi X, \Phi Y]+\Phi^{2}[X, Y]-\Phi[\Phi X, Y]-\Phi[X, \Phi Y]-$ тензор Нейенхейса структурного оператора $\Phi$.

Нормальную почти контактную метрическую структуру, структурньй ковектор которой удовлетворяет тождеству $\nabla_{X}(\eta) Y+\nabla_{Y}(\eta) X=0$, назовем $\mathscr{C} \mathscr{N} \mathscr{K}$-структурой.

Известно [1], что задание почти контактной метрической структуры на многообразии $M^{2 n+1}$ определяет в модуле $\varkappa(M)$ пару взаимно-дополнительных проекторов: $m=\xi \otimes \eta$, $l=\mathrm{id}-\xi \otimes \eta$. Известно также, что $l=-\Phi^{2},\langle\Phi l X, \Phi l Y\rangle=\langle l X, l Y\rangle, X, Y \in \varkappa(M)$. Это означает, что на распределении $\mathscr{L}=\operatorname{Im} \Phi$ пара $\left\{\Phi|\mathscr{L}, g|_{\mathscr{L}}\right\}$ определяет почти эрмитову структуру; следовательно, $\mathscr{L}$ можно рассматривать как эрмитово векторное расслоение над $M$ с метрикой $H(X, Y)=\langle X, Y\rangle+\sqrt{-1}\langle X, \Phi Y\rangle, X, Y \in \varkappa(M)$.

В расслоении $\mathscr{L}^{\mathbb{C}}=\mathscr{L} \otimes \mathbb{C}$ над $M$ естественно определены взаимно-дополнительные проекторы $\sigma=\frac{1}{2}\left(\mathrm{id}-\left.\sqrt{-1} \Phi\right|_{\mathscr{L}}\right), \bar{\sigma}=\frac{1}{2}(\mathrm{id}+\sqrt{-1} \Phi \mathscr{L})$ (здесь под оператором $\left.\Phi\right|_{\mathscr{L}}$, вообще говоря, понимается его комплексификация [5] $\left.\Phi^{\mathbb{C}}=\left(\left.\Phi\right|_{\mathscr{L}}\right) \otimes \mathrm{id}_{\mathbb{C}}\right)$, а также оператор $\tau$ естественного сопряжения, причем очевидно, что на $\mathscr{L}$ справедливо соотношение $\bar{\sigma}=\tau \circ \sigma[1]$.

Задание проекторов $\sigma$ и $\bar{\sigma}$ равносильно распаданию модуля $\mathscr{L}^{\mathbb{C}}$ в прямую сумму двух подмодулей $\mathscr{D}$ и $\overline{\mathscr{D}}$ - образов этих проекторов. Легко видеть, что $\mathscr{D}$ и $\overline{\mathscr{D}}$ являются собственньми подмодулями оператора $\Phi$ с собственньми значениями $\sqrt{-1}$ и $-\sqrt{-1}$ соответственно.

Пусть $p \in M$. Выберем в $\mathscr{L}_{p}$ репер $\left\{p, e_{1}, \ldots, e_{n}\right\}$, унитарньй относительно метрики $H$. Построим репер $\left\{p, \varepsilon_{0}, \varepsilon_{1}, \ldots, \varepsilon_{n}, \varepsilon_{\widehat{1}}, \ldots, \varepsilon_{\widehat{n}}\right\}$ модуля $\mathscr{L}_{p}^{\mathbb{C}} \oplus \mathfrak{M}_{p}$, где $\mathfrak{M}_{p}=\operatorname{Im}\left(m_{p}\right)$, положив $\varepsilon_{0}=\xi, \varepsilon_{a}=\sigma\left(e_{a}\right), \varepsilon_{\widehat{a}}=\bar{\sigma}\left(e_{a}\right)$, где условимся считать, что индексы $i, j, k, s$ пробегают значения от 0 до $2 n$, а индексы $a, b, c, d, g$ - значения от 1 до $n, \widehat{a}=a+n$. Заметим, что $\Phi\left(\varepsilon_{a}\right)=\sqrt{-1} \varepsilon_{a}, \Phi\left(\varepsilon_{\widehat{a}}\right)=-\sqrt{-1} \varepsilon_{\widehat{a}}, \Phi\left(\varepsilon_{0}\right)=\Phi(\xi)=0$.

Репер $\left\{p, \varepsilon_{0}, \varepsilon_{1}, \ldots, \varepsilon_{n}, \varepsilon_{\widehat{1}}, \ldots, \varepsilon_{\widehat{n}}\right\}$ называется репером, адаптированным струкmуре, или $A$-репером [6]. Известно [1], что задание почти контактной метрической структуры на многообразии $M^{2 n+1}$ равносильно заданию $G$-структуры на пространстве расслоения всех реперов на многообразии $M^{2 n+1}$ со структурной группой $G=$ $U(n) \times\{1\} . A$-реперы характеризуются тем, что матрицы тензоров $\Phi$ и $g$ в них имеют вид

$$
\left(\Phi_{j}^{i}\right)=\left(\begin{array}{ccc}
0 & 0 & 0 \\
0 & \sqrt{-1} I_{n} & 0 \\
0 & 0 & -\sqrt{-1} I_{n}
\end{array}\right), \quad\left(g_{i j}\right)=\left(\begin{array}{ccc}
1 & 0 & 0 \\
0 & 0 & I_{n} \\
0 & I_{n} & 0
\end{array}\right)
$$

где $I_{n}$ - единичная матрица порядка $n$.

Пусть $\omega$ - форма смещения, $\varphi$ - форма римановой связности $\nabla$ на пространстве присоединенной $G$-структуры. На пространстве этой $G$-структуры форма связности $\varphi$ задается формами $\left\{\omega_{j}^{i}\right\}$, а форма смещения $\omega$ - формами $\left\{\omega^{i}\right\}$. На пространстве присоединенной $G$-структуры полная группа структурных уравнений $\mathscr{C} \mathscr{N} \mathscr{K}$-многообразия 
имеет вид [7]

$$
\begin{gathered}
d \omega=2 B_{a}{ }^{b} \omega^{a} \wedge \omega_{b}, \quad d \omega^{a}=\Theta_{b}^{a} \wedge \omega_{b}+B^{a b}{ }_{c} \omega^{c} \wedge \omega_{b} \\
d \omega_{a}=-\Theta_{a}^{b} \wedge \omega_{b}+B_{a b}{ }^{c} \omega^{b} \wedge \omega_{c} \\
d \Theta_{b}^{a}=\Theta_{g}^{a} \wedge \Theta_{b}^{g}+A_{b g}^{a c} \omega^{g} \wedge \omega_{c}+A_{b g c}^{a} \omega^{g} \wedge \omega^{c}+A_{b}^{a g c} \omega_{g} \wedge \omega_{c} \\
d B^{a b}{ }_{c}=B^{g b}{ }_{c} \Theta_{g}^{a}+B^{a g}{ }_{c} \Theta_{g}^{b}-B^{a b}{ }_{g} \Theta_{a}^{g}+B^{a b}{ }_{c g} \omega^{g}+B^{a b}{ }_{c}{ }^{g} \omega_{g} \\
d B_{a b}{ }^{c}=-B_{g b}{ }^{c} \Theta_{a}^{g}-B_{a g}{ }^{c} \Theta_{b}^{g}+B_{a b}{ }^{g} \Theta_{g}^{a}+B_{a b}{ }^{c}{ }_{g} \omega^{g}+B_{a b}{ }^{c g} \omega_{g},
\end{gathered}
$$

где $\Theta_{b}^{a}=\omega_{b}^{a}-B_{b}{ }^{a} \omega$. Причем

$$
\begin{gathered}
\omega^{\widehat{a}}=\omega_{a}, \quad B_{a}{ }^{b}=\sqrt{-1} \Phi_{0, \widehat{b}}^{\widehat{a}}, \quad \bar{B}_{a}{ }^{b}=-B_{b}{ }^{a}, \\
B_{a b}{ }^{c}=-\frac{\sqrt{-1}}{2} \Phi_{b, \widehat{c}}^{\widehat{a}}, \quad \bar{B}_{a b}{ }^{c}=-B^{a b}{ }_{c}, \quad B_{a b}{ }^{c}=-B_{b a}{ }^{c}, \\
\bar{A}_{b d}^{a c}=A_{a c}^{b d}, \quad \bar{A}_{b d c}^{a}=-\bar{A}_{a}^{b d c}, \\
\bar{B}_{a b}{ }^{c d}=-B^{a b}{ }_{c d}, \quad \bar{B}_{a b}{ }^{c}{ }_{d}=-B^{a b}{ }_{c}{ }_{d}, \quad B_{a b}{ }^{c d}=-B_{b a}{ }^{c d}, \quad B_{a b}{ }^{c}{ }_{d}=-B_{b a}{ }^{c}{ }_{d} .
\end{gathered}
$$

Соотношения (2) представляют собой системы функций на пространстве присоединенной $G$-структуры, служащие компонентами соответствующих тензоров. Отметим также существование структурного тождества [7]

$$
A_{[a b]}^{c d}=B_{[a b]}^{c d}-B_{[a}^{c h} B_{b] h}{ }^{d}
$$

Скобки $[\cdot]$ и $(\cdot)$ означают соответственно операции альтернирования и симметризации объекта по заключенным в них индексам.

Рассматривая второе уравнение Картана связности $\nabla$ [8], получаем спектр тензора Римана-Кристоффеля, т.е. выражение его компонент на пространстве присоединенной G-структуры [7]:

$$
\begin{gathered}
R_{b c d}^{a}=2 A_{b c d}^{a}, \quad R_{b c \widehat{d}}^{a}=A_{b c}^{a d}-2 B_{b}{ }^{a} B_{c}{ }^{d}-B_{c}{ }^{a} B_{b}{ }^{d}+B^{a h}{ }_{c} B_{h b}{ }^{d}, \\
R_{b c 0}^{a}=-C_{b c}^{a}, \quad R_{b c d}^{\widehat{a}}=0, \quad R_{b c d}^{0}=0, \\
R_{b c \widehat{a}}^{\widehat{a}}=-B_{a b}{ }_{b c}{ }_{b c}-B_{a b}{ }^{h} B_{h c}{ }^{d}, \quad R_{b \widehat{c} \widehat{d}}^{\widehat{a}}=2\left(B_{a}{ }^{[c} B_{b}{ }^{d]}+B_{a b}{ }^{[c d]}\right), \\
R_{b c 0}^{\widehat{a}}=0, \quad R_{b c 0}^{0}=0, \quad R_{b \widehat{c} \widehat{d}}^{0}=0, \quad R_{b \widehat{c} 0}^{0}=-B_{b}{ }_{b}{ }_{b h}{ }^{c} .
\end{gathered}
$$

Напомним [9], что почти контактная метрическая структура $\{\Phi, \xi, \eta, g=\langle\cdot, \cdot\rangle\}$ назьвается квазисасакиевой, если она нормальная и ее фундаментальная 2 -форма $\Omega(X, Y)=$ $\langle X, \Phi Y\rangle, X, Y \in \varkappa(M)$, замкнута. На пространстве присоединенной $G$-структуры первая группа структурных уравнений квазисасакиева многообразия в принятых обозначениях (2) имеет вид [10]

$$
d \omega=2 B_{b}{ }^{a} \omega^{b} \wedge \omega_{a}, \quad d \omega^{a}=\Theta_{b}^{a} \wedge \omega^{b}, \quad d \omega_{a}=-\Theta_{a}^{b} \wedge \omega_{b}
$$


ОПРЕДЕЛЕниЕ 2 [6]. Пусть $M-(2 n+1)$-мерное почти контактное многообразие. Говорят, что оно удовлетворяет аксиоме $\Phi$-голоморфных плоскостей, если $\forall p \in M$, $\forall L \subset T_{p}(M)$, где $L$ - двумерная $\Phi$-инвариантная площадка, ортогональная $\xi, \Phi(L) \subset L$, $\exists N \subset M$ - вполне геодезическое $\Phi$-инвариантное подмногообразие, т.е. $\Phi(N)=N$ такое, что $p \in N \Longrightarrow T_{p}(N)=L$.

Пусть $M^{2 n+1}-\mathscr{C} \mathscr{N} \mathscr{K}$-многообразие, удовлетворяющее аксиоме $\Phi$-голоморфных плоскостей, $p \in M-$ произвольная точка многообразия $M, T_{p}(M)$ - касательное пространство в этой точке, $L \subset T_{p}(M)-\Phi$-инвариантное пространство, ортогональное вектору $\xi$.

Пусть $\left\{p, \xi, \varepsilon_{1}, \ldots, \varepsilon_{n}, \varepsilon_{\hat{1}}, \ldots, \varepsilon_{\widehat{n}}\right\}$ - произвольньй $A$-репер в пространстве $T_{p}(M)$, $(p, \beta, \bar{\beta})-A$-репер в $T_{p}(N)$. Если $j: T_{p}(N) \rightarrow T_{p}(M)$ - естественное вложение, то оно задается формулами

$$
\begin{aligned}
& \beta=G^{a} \varepsilon_{a}, \\
& \bar{\beta}=G_{a} \varepsilon_{\widehat{a}} .
\end{aligned}
$$

В силу вешественности $j$ имеем $\overline{G^{\widehat{a}}}=G_{a}$. Соотношения, двойственные соотношениям (6), задаются формулами

$$
\begin{aligned}
& \omega^{a}=G^{a} \theta, \\
& \omega_{a}=G_{a} \theta,
\end{aligned}
$$

где $(p, \theta, \bar{\theta})$ - корепер, дуальный $A$-реперу $(p, \beta, \bar{\beta})$.

Продифференцируем внешним образом соотношение $\left(7_{1}\right)$ :

$$
d \omega^{a}=d G^{a} \wedge \theta+G^{a} d \theta .
$$

С учетом формул $(1),\left(7_{1}\right),\left(7_{2}\right)$ последнее выражение можно записать в виде

$$
d \theta=G_{a}\left(G^{b} \Theta_{b}^{a}-B_{c}^{a b} G^{c} G_{b} \bar{\theta}-d G^{a}\right) \wedge \theta .
$$

Рассмотрим в выражении (8) слагаемое $-B^{a b}{ }_{c} G^{c} G_{a} G_{b} \bar{\theta}$. В силу кососимметричности тензора $B^{a b}{ }_{c}$ по индексам $a$ и $b$ имеем

$$
-B^{a b}{ }_{c} G^{c} G_{a} G_{b} \bar{\theta}=B^{b a}{ }_{c} G^{c} G_{b} G_{a} \bar{\theta}=B^{a b}{ }_{c} G^{c} G_{a} G_{b} \bar{\theta} .
$$

Следовательно, $B^{a b}{ }_{c} G^{c} G_{a} G_{b} \bar{\theta}=0$. С учетом этого тождества выражение (8) принимает вид

$$
d \theta=G_{a}\left(G^{b} \Theta_{b}^{a}-d G^{a}\right) \wedge \theta .
$$

Обозначим $\psi=G^{b} \Theta_{b}^{a} G_{a}-G_{a} d G^{a}$. Тогда выражение (9) запишется в виде

$$
d \theta=\psi \wedge \theta
$$

С учетом тождеств (10) и (8) будем иметь

$$
\left(G^{b} \Theta_{b}^{a}-d G^{a}-B_{c}^{a b} G^{c} G_{b} \bar{\theta}-G^{a} \psi\right) \wedge \theta=0 .
$$

Отсюда в силу леммы Картана получаем

$$
G^{b} \Theta_{b}^{a}-d G^{a}-B_{c}^{a b} G^{c} G_{b} \bar{\theta}-G^{a} \psi=\Delta^{a} \theta,
$$

или это можно записать в виде

$$
G^{b} \Theta_{b}^{a}-d G^{a}-G^{a} \psi=\Delta^{a} \theta+B^{a b}{ }_{c} G^{c} G_{b} \bar{\theta} .
$$


ТЕорема 1. $\mathscr{C} \mathscr{N} \mathscr{K}$-многообразие $M$, удовлетворяющее аксиоме $\Phi$-голоморфных плоскостей, является квазисасакиевым.

ДокАЗАТЕльСтво. Пусть многообразие $M$ удовлетворяет аксиоме $\Phi$-голоморфных плоскостей. Тогда вторая квадратичная форма этого многообразия равна нулю, т.е. из равенства (11) имеем

$$
\Delta^{a} \theta+B^{a b}{ }_{c} G^{c} G_{b} \bar{\theta}=0 .
$$

В силу линейной независимости форм $\theta$ и $\bar{\theta}$ имеем

$$
\begin{gathered}
\Delta^{a}=0, \\
B^{a b}{ }_{c} G^{c} G_{b}=0 .
\end{gathered}
$$

Аналогично найдем

$$
\begin{gathered}
\Delta_{a}=0, \\
B_{a b}{ }^{c} G_{c} G^{b}=0 .
\end{gathered}
$$

Заметим, что тождества $\left(12_{2}\right)$ и $\left(13_{2}\right)$ должны вьполняться тождественно относительно $G^{i}$; следовательно,

$$
B^{a b}{ }_{c}=0, \quad B_{a b}{ }^{c}=0 .
$$

Равенства (14) означают, что первая групша структурных уравнений (1) $\mathscr{C} \mathscr{N} \mathscr{K}$-многообразия, удовлетворяюшего аксиоме $\Phi$-голоморфных плоскостей, имеет вид

$$
d \omega=2 B_{a}{ }^{b} \omega^{a} \wedge \omega_{b}, \quad d \omega^{a}=\Theta_{b}^{a} \wedge \omega^{b}, \quad d \omega_{a}=-\Theta_{a}^{b} \wedge \omega_{b} .
$$

Сравнивая эту группу уравнений с группой уравнений (5), получаем, что многообразие $M$ является квазисасакиевым.

Известно [11], что квазисасакиево многообразие удовлетворяет аксиоме $\Phi$-голоморфных плоскостей тогда и только тогда, когда верно равенство

$$
A_{b d}^{a g}=\frac{A}{n(n+1)} \widehat{\delta}_{b d}^{a g}
$$

где $A$ - результат свертки тензора $A_{d}^{g}=A_{(d a)}^{a g}$ по индексам $g$ и $d, \widehat{\delta}_{b d}^{a g}=\delta_{b}^{a} \delta_{d}^{g}+\delta_{b}^{g} \delta_{d}^{a}$. Тогда с учетом теоремы 1 справедлива следующая теорема.

Теорема 2. $\mathscr{C} \mathscr{N} \mathscr{K}$-многообразие $M^{2 n+1}$ удовлетворяет аксиоме $\Phi$-голомор ных плоскостей тогда и только тогда, когда оно квазисасакиево и выполняется равенство (15).

Напомним [2], что почти контактное метрическое многообразие $M$ называется многообразием точечно постоянной $\Phi$-голоморфной секиионной кривизны, если

$$
\forall X \in \mathscr{L} \Longrightarrow\langle R(X, \Phi X) X, \Phi X\rangle=c\|X\|^{4}, c \in C^{\infty}(M) .
$$

На пространстве присоединенной $G$-структуры это равенство запишется в виде

$$
R_{i j k s} X^{i}(\Phi X)^{j} X^{k}(\Phi X)^{s}=2 c \widehat{\delta}_{g d}^{a b} X^{g} X^{d} X_{a} X_{b}
$$


ТЕОремА 3. $\mathscr{C} \mathscr{N} \mathscr{K}$-многообразие $M$ является многообразием точечно постоянной $\Phi$-голоморфной секиионной кривизны тогда и только тогда, когда выражение для $A_{a b}^{g d}$ имеет вид

$$
A_{a b}^{g d}=\frac{c}{2} \widehat{\delta}_{a b}^{g d}+3 B_{(a}^{(g} B_{b)}{ }^{d)}+B_{a b}{ }^{[g d]}-B_{h a}{ }^{d} B_{b}^{g h} .
$$

ДокАЗАТЕЛЬСтво. Пусть $M-\mathscr{C} \mathcal{N} \mathscr{K}$-многообразие точечно постоянной $\Phi$-голоморфной секционной кривизны. Расписывая подробно тождество (16) с учетом соотношений (4) и тождеств $(\Phi X)^{a}=\sqrt{-1} X^{a},(\Phi X)^{\widehat{a}}=-\sqrt{-1} X^{\widehat{a}}$, получим

$$
\left(A_{(a b)}^{(g d)}+B_{h(a}^{(g} B_{b)}^{d) h}-3 B_{(a}^{(g} B_{b)}{ }^{d)}\right) X^{a} X^{b} X_{g} X_{d}=\frac{c}{2} \widehat{\delta}_{a b}^{g d} X^{a} X^{b} X_{g} X_{d}
$$

Введем в рассмотрение 4-форму $C(X, Y, Z, W)$ формулой

$$
\begin{gathered}
C(X, Y, Z, W)=\left(A_{(a b)}^{(g d)}+B_{h(a}{ }^{(g} B_{b) h}^{d) h}-3 B_{(a}{ }^{(g} B_{b)}{ }^{d)}-\frac{c}{2} \widehat{\delta}_{a b}^{g d}\right) X^{a} Y^{b} Z_{g} W_{d} \\
\forall X, Y, Z, W \in \mathscr{L} .
\end{gathered}
$$

Легко показать, что 4-форма $C(X, Y, Z, W)$ обладает свойствами:

1) $C(X, X, X, X)=0$

2) линейна по первой паре аргументов;

3) антилинейна по второй паре аргументов.

Опираясь на эти свойства, нетрудно найти

$$
C(X, Y, Z, W)=0 \quad \forall X, Y, Z, W \in \mathscr{L} .
$$

Из этого следует справедливость равенства

$$
A_{(a b)}^{(g d)}=\frac{c}{2} \widehat{\delta}_{a b}^{g d}+3 B_{\left(a^{(g} B_{b)} d\right)}-B_{h(a}^{(g} B_{b)}^{d) h} .
$$

Запишем объект $A_{a b}^{g d}$ в форме

$$
A_{a b}^{g d}=A_{[a b]}^{[g d]}+A_{(a b)}^{[g d]}+A_{[a b]}^{(g d)}+A_{(a b)}^{(g d d)} .
$$

Из соотношений (3), (18), (19) с учетом кососимметричности $B_{a b}{ }^{g d}, B_{g d}^{a b}$ по индексам $a$ и $b$ получим выражение (17).

Обратное очевидно.

Теорема 4. Пусть $M-\mathscr{C} \mathscr{N} \mathscr{K}$-многообразие точечно постоянной $\Phi$-голомор ной секиионной кривизны. Многообразие $M$ удовлетворяет аксиоме $\Phi$-голоморфных плоскостей тогда и только тогда, когда $M$ локально әквивалентно одному из следующих многообразий:
1) $\mathbb{C}^{n} \times \mathbb{R}$
2) $\mathbb{C P}^{n} \times \mathbb{R}$
3) $\mathbb{C} D^{n} \times \mathbb{R}$;
4) $M^{2} \times \mathbb{R}$ 
5) нечетномерной сфере, снабженной канонической сасакиевой структурой или структурой, полученной из канонической преобразованием D्D-гомотетuи [6], [12];

6) нечетномерному аффинному пространству, снабженному канонической сасакиевой структурой постоянной Ф-голоморфной секционной кривизHbl -3

7) нечетномерной сфере, снабженной сасакиевой структурой, полученной из канонической $\mathscr{D}$-инверсией [6].

ДокАЗАТЕЛЬСТво. Пусть $M-\mathscr{C} \mathscr{N} \mathscr{K}$-многообразие точечно постоянной $\Phi$-голоморфной секционной кривизны, удовлетворяющее аксиоме $\Phi$-голоморфных плоскостей. Тогда с учетом теоремы 1 и теоремы 3 оно квазисасакиево и справедливы равенства (15), (17). Но для квазисасакиева многообразия тензоры $B_{a b}{ }^{g}, B^{a b}{ }_{g}, B_{a b}{ }^{g d}$ нулевые; следовательно, равенства (15) и (17) будут равносильны следующему:

$$
\frac{c}{2} \widehat{\delta}_{a b}^{g d}+3 B_{a}^{g} B_{b}{ }^{d}=\frac{A}{n(n+1)} \widehat{\delta}_{a b}^{g d},
$$

откуда находим

$$
B_{a}{ }^{d} B_{b}{ }^{g}+B_{b}{ }^{d} B_{a}{ }^{g}=\frac{2}{3} \widehat{\delta}_{a b}^{g d}\left(\frac{A}{n(n+1)}-\frac{c}{2}\right) .
$$

Обозначим

$$
k=\frac{2}{3}\left(\frac{A}{n(n+1)}-\frac{c}{2}\right) .
$$

Тогда предыдущее равенство принимает вид

$$
B_{a}{ }^{d} B_{b}{ }^{g}+B_{b}{ }^{d} B_{a}{ }^{g}=k \widehat{\delta}_{a b}^{g d}
$$

Поскольку $\bar{B}_{b}{ }^{a}=-B_{a}{ }^{b}$, то оператор $B: \mathscr{D}_{\Phi}^{\sqrt{-1}} \rightarrow \mathscr{D}_{\Phi}^{\sqrt{-1}}$ косоэрмитов и, значит, в подходящем $A$-репере $\left(p, \varepsilon_{a}, \varepsilon_{\widehat{a}}\right)$ приводится к диагональному виду относительно базиса $\left\{\varepsilon_{1}, \ldots, \varepsilon_{n}\right\}$ пространства $\left(\mathscr{D}_{\Phi}^{\sqrt{-1}}\right)_{p}$. В этом базисе выражение для $B_{a}{ }^{b}$ принимает вид $B_{a}{ }^{b}=B_{b} \delta_{a}^{b}$. Тогда соотношение $(20)$ в этом базисе записьвается в форме

$$
B_{d} B_{g} \widehat{\delta}_{a b}^{g d}=k \widehat{\delta}_{a b}^{g d}
$$

Положив здесь $a=b=g=d$, получим

$$
\left(B_{a}\right)^{2}=k
$$

Полагая теперь $a=d, b=g, a \neq b$, получим

$$
B_{a} \cdot B_{b}=k \text {. }
$$

В частности, из $(21),(22)$ следует, что $\left(B_{a}\right)^{2} \cdot B_{b}=k \cdot B_{a}$, т.е. $k \cdot B_{b}=k \cdot B_{a}$, т.е. $k\left(B_{b}-B_{a}\right)=0$.

1) Если $k=0$, то из соотношения (21) следует, что $B_{1}=B_{2}=\cdots=B_{n}=0$, откуда в силу соотношения $B_{a}{ }^{b}=B_{b} \delta_{a}^{b}$ получаем $B_{a}{ }^{b}=0$. Это означает вьполнение равенств $\nabla \Phi=\nabla \eta=\nabla \xi=0$. Значит, многообразие $M$ косимплектическое. 
2) Если $k \neq 0$, то $B_{a}=B_{b}, a \neq b$. При этом, очевидно, $B_{a}=B_{b}=\sqrt{k}$. Итак, $B_{a}{ }^{b}=\sqrt{k} \delta_{a}^{b}$. Заметим, что при комплексном сопряжении этого равенства, оно принимает вид

$$
-B_{b}{ }^{a}=\overline{\sqrt{k}} \delta_{b}^{a}
$$

т.е. $\overline{\sqrt{k}}=-\sqrt{k}$. Иначе говоря, $\sqrt{k}-$ число чисто мнимое, т.е. $k<0$. Пусть $k=-\gamma^{2}$, где $\gamma \in \mathbb{R}$. Тогда

$$
B_{a}^{b}=\sqrt{-1} \gamma \delta_{a}^{b}
$$

В этом случае имеем

$$
d \eta=d \omega=2 \sqrt{-1} \gamma \delta_{a}^{b} \omega^{a} \wedge \omega_{b}=2 \sqrt{-1} \gamma \omega^{a} \wedge \omega_{b}=-\gamma \Omega
$$

Продифференцируем внешним образом соотношение $-d \omega=\gamma \Omega$. Получаем

$$
d \gamma \wedge \Omega+\gamma d \Omega=0
$$

С учетом теоремы $1 \mathscr{C} \mathscr{N} \mathscr{K}$-многообразие, удовлетворяющее аксиоме плоскостей, квазисасакиево, т.е. его фундаментальная 2-форма $\Omega$ замкнута; следовательно, имеем

$$
d \gamma \wedge \Omega=0
$$

т.е.

$$
d \gamma \wedge \omega^{a} \wedge \omega_{a}=0
$$

Пусть $d \gamma=\gamma_{b} \omega^{b}+\gamma^{b} \omega_{b}+\gamma^{0} \omega$. Тогда с учетом предыдущего равенства находим

$$
\gamma_{b} \omega^{b} \wedge \omega^{a} \wedge \omega_{a}+\gamma^{b} \omega_{b} \wedge \omega^{a} \wedge \omega_{a}+\gamma^{0} \omega \wedge \omega^{a} \wedge \omega_{a}=0
$$

Отсюда в силу линейной независимости базисных форм находим

$$
\gamma_{b}=\gamma^{b}=\gamma^{0}=0
$$

т.е. $d \gamma=0$; значит, $\gamma=$ const.

Совершим гомотетическое преобразование метрического тензора структуры $\{\Phi, \xi$, $\eta, g\}$, положив $\widetilde{g}=\gamma^{2} g$. Тогда в новой метрике $\|\xi\|_{\widetilde{g}}^{2}=\gamma^{2}$. Выбрав $\widetilde{\xi}=\frac{1}{\gamma} \xi$, получим $\|\widetilde{\xi}\|_{\widetilde{g}}^{2}=1$. Однако $\eta(\widetilde{\xi})=\frac{1}{\gamma} \eta(\xi)$. Поэтому положим $\widetilde{\eta}=\gamma \eta$. Тогда $\widetilde{\eta}(\widetilde{\xi})=1$. Наконец, $\widetilde{\Omega}(X, Y)=\widetilde{g}(X, \Phi Y)=\gamma^{2} g(X, \Phi Y)=\gamma^{2} \Omega(X, Y)$. Итак, для новой почти контактной метрической структуры $\{\Phi, \widetilde{\xi}, \widetilde{\eta}, \widetilde{g}\}$, где $\widetilde{\xi}=\frac{1}{\gamma} \xi, \widetilde{\eta}=\gamma \eta, \widetilde{g}=\gamma^{2} g$, имеем $d \widetilde{\eta}=\gamma d \eta=\gamma^{2} \Omega=\widetilde{\Omega}$, т.е. структура $\{\Phi, \widetilde{\xi}, \widetilde{\eta}, \widetilde{g}\}$ сасакиева.

Переход от структуры $\{\Phi, \xi, \eta, g\}$ к структуре $\{\Phi, \widetilde{\xi}, \widetilde{\eta}, \widetilde{g}\}$ называется гомотетическим преобразованием структуры. Значит, наша структура гомотетична сасакиевой.

Итак, если $\mathscr{C} \mathscr{N} \mathscr{K}$-многообразие $M$ точечно постоянной $\Phi$-голоморфной секционной кривизны удовлетворяет аксиоме $\Phi$-голоморфных плоскостей, то $M$ либо косимплектическое, либо с точностью до гомотетического преобразования структуры сасакиево.

Известно [13], что косимплектическое многообразие постоянной $\Phi$-голоморфной секционной кривизны локально эквивалентно произведению келерова многообразия постоянной $\Phi$-голоморфной секционной кривизны на вешественную прямую. В свою очередь, 
келерово многообразие постоянной $\Phi$-голоморфной секционной кривизны локально эквивалентно одному из следующих многообразий [14], [15]:

а) $\mathbb{C}^{n}$ - комплексному евклидовому пространству;

б) $\mathbb{C P}^{n}$ - комплексному проективному пространству;

в) $\mathbb{C} D^{n}$ - комплексному гиперболическому пространству;

г) $M^{2}$ - двумерному многообразию, снабженному канонической келеровой структурой.

С другой стороны, в работах [1], [12] приведена классификация сасакиевых многообразий точечно постоянной $\Phi$-голоморфной секционной кривизны, удовлетворяющих аксиоме $\Phi$-голоморфных плоскостей. Всякое такое многообразие эквивалентно одному из многообразий, перечисленных в утверждении теоремы 4.

Обратное немедленно следует из того, что многообразия, перечисленные в списке теоремы 4, являются косимплектическими либо сасакиевыми пространственньми формами и, следовательно, как хорошо известно, имеют постоянную $\Phi$-голоморфную секционную кривизну и удовлетворяют аксиоме $\Phi$-голоморфных плоскостей [6].

\section{СПИСОК ЦИТИРОВАННОЙ ЛИТЕРАТУРЫ}

[1] Кириченко В. Ф. Методы обобщенной почти эрмитовой геометрии в теории почти контактных многообразий // Итоги науки и техники. Проблемы геометрии. Т. 18. М.: ВИНИТИ, 1986. C. $25-71$.

[2] Ishihara I. Anti-invariant submanifolds of a Sasakian space form // Kodai Math. J. 1979. V. 2. P. 171-186.

[3] Tanno S. Sasakian manifolds with constant $\varphi$-holomorphic sectional curvature // Tôhoku Math. J. 1969. V. 21. № 3. P. 501-507.

[4] Goldberg S., Jano K. Integrability of almost cosymplectic structures // Pacific J. Math. 1969. V. 31. № 2. P. 373-382.

[5] Ефимов Н. В., Розендорн Э. Р. Линейная алгебра и многомерная геометрия. М.: Наука, 1970.

[6] Кириченко В.Ф. Аксиома Ф-голоморфных плоскостей в контактной метрической геометрии // Изв. АН СССР. Сер. матем. 1984. Т. 48. № 4. С. 711-734.

[7] Волкова Е. С. О геометрии нормальных многообразий киллингова типа // Деп. ВИНИТИ РАН № 2111-В96. М.: ВИНИТИ, 1996.

[8] Кобалси Ш., Номидзу К. Основы дифференциальной геометрии. Т. 1, 2. М.: Наука, 1981.

[9] Blair D.E. The theory of Quasi-Sasakian structures // J. Diff. Geometry. 1967. V. 1. P. 331-345.

[10] Рустанов А.Р. О геометрии квазисасакиевых многообразий // Деп. ВИНИТИ РАН № 962-В94. М.: ВИНИТИ, 1994.

[11] Рустанов А.Р. Аксиома Ф-голоморфных плоскостей для квазисасакиевых многообразий // Научные труды МПГУ им. В. И. Ленина. М., 1994. С. 39-45.

[12] Kiritchenko V.F. Classification des varietés presque sasakiennes satisfaisant à l'axiome des plans $\Phi$-holomorphes // C. R. Acad. Sci. Sér. I. 1982. V. 295. №13. P. 739-742.

[13] Kiritchenko V.F. Sur la géometrie des varietés approximativement cosymplectiques // C. R. Acad. Sci. Sér. I. 1982. V. 295. № 12. P. 673-676.

[14] Hawley N.S. Constant holomorphic sectional curvature // Canad. Math. J. 1953. V. 5. P. 53-56.

[15] Igusa J. On the structure of a certain class of Kähler manifolds // Nat. Sc. Rep. Ochanomizu Univ. 1980. V. 31. № 1. P. 42-54. 